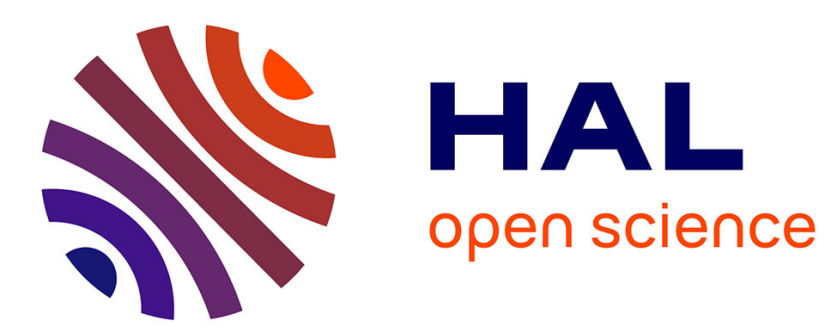

\title{
Combined Structure-Control Optimal Design of the Stewart-Gough Robot.
}

Fabian Andres Lara-Molina, Edson Koroishi, Didier Dumur

\section{To cite this version:}

Fabian Andres Lara-Molina, Edson Koroishi, Didier Dumur. Combined Structure-Control Optimal Design of the Stewart-Gough Robot.. 12th LARS Latin American Robotics Symposium 2015, Oct 2015, Uberlândia -MG, Brazil. 10.1109/lars-sbr.2015.26 . hal-01258935

\section{HAL Id: hal-01258935 \\ https://hal-centralesupelec.archives-ouvertes.fr/hal-01258935}

Submitted on 12 Mar 2020

HAL is a multi-disciplinary open access archive for the deposit and dissemination of scientific research documents, whether they are published or not. The documents may come from teaching and research institutions in France or abroad, or from public or private research centers.
L'archive ouverte pluridisciplinaire HAL, est destinée au dépôt et à la diffusion de documents scientifiques de niveau recherche, publiés ou non, émanant des établissements d'enseignement et de recherche français ou étrangers, des laboratoires publics ou privés. 


\section{Combined Structure-Control Optimal Design of the Stewart-Gough Robot}

\author{
F. A. Lara-Molina and E. H. Koroishi \\ Department of Mechanical Engineering \\ Federal Technological University of Paraná (UTFPR) \\ Cornélio Procópio-PR, Brazil \\ Email: \{fabianmolina,edsonh $\} @$ utfpr.edu.br
}

\author{
Didier Dumur \\ Control Department \\ LSS - Centrale Supélec \\ Gif sur Yvette Cedex F-91192, France \\ Email: Didier.Dumur@centralesupelec.fr
}

\begin{abstract}
This paper aims at introducing an optimal design methodology for the Stewart Platform robot controlled by a PD position controller. This optimal design method intends to maximize the positioning accuracy by using metaheuristic optimization methods. The structure design variables of the mechanism combined with the gains of the controller are the structure-control design variables. The structure-control design variables are considered simultaneously in the optimal design method. A sensitivity analysis is performed to evaluate the effect of the structure-control design variables, especially of structure variables, on the positioning accuracy. Simulation results demonstrate that the proposed design procedure is effective to increase the position accuracy, as well as the closed loop dynamics performance of the robot is improved.
\end{abstract}

\section{INTRODUCTION}

The optimal design of parallel robots aims at determining a set of design variables to satisfy an optimal performance criterion. Many works have addressed the optimal design of parallel robots by using different approaches in order to optimize a desired performance criterion.

The growing number of new applications of parallel robots demands to increase the positioning accuracy. The structure design of the mechanism and the design of the control system have been addressed separately. The structure design of the mechanism has been figured up to obtain high kinematic performance, some works have addressed this problem by maximizing kinematic accuracy [1]-[3]. The design of the controller intends to improve the dynamic behavior to perform a specific task, thus, some works have applied advanced position controllers to enhance the positioning accuracy [4][6].

The mechatronic design has been used as an alternative to optimal design of parallel robots, this methodology takes into account structural-control design variables of the system simultaneously during the design procedure. Mechatronic design has been applied to parallel robots [7]-[9]. Villareal-Cervantes et al. (2009) [7] proposed structure-control mechatronic design of the planar parallel robot. Silva et al. (2013) [8] figured up the optimal design of a pick-and-place robotic system by using mechatronic design concepts. Villareal-Cervantes et al. (2013) [9] developed a robust formulation for the mechatronic design of planar parallel robot. All the aforementioned works are concerned with planar parallel robots. Nevertheless, some applications require high-performance positioning systems with spatial parallel mechanism.
In this contribution, we propose an optimization of structure-control design variables simultaneously to maximize position tracking accuracy of the Stewart-Gough robot through the design method. A sensitivity analysis, based on variance decomposition, is performed to quantify the effect of structure and control variables on position tracking accuracy. The optimization problem is solved by using metaheuristic optimization methods.

The remain of this paper is organized in several sections. Section II presents the robot modeling to introduce the structure-control design variables and the objective function. In section III, a sensitivity analysis method is presented to quantify the effect of the design variables on the objective function. In section IV, the optimization problem to solve the optimal design is presented. Section $\mathrm{V}$ gives the simulation results. Finally, section VI enumerates some conclusions.

\section{RoBOt MODELING}

The 6-UPS Stewart-Gough manipulator has six identical legs connecting the fixed base to the movable platform by universal joints denoted by $U$ at points $B_{i}$ and spherical joints denoted by $S$ at points $P_{i}$ (for $i=1, \ldots, 6$ ), respectively. Both the universal and the spherical joints are passive. Each leg has an upper and a lower member connected by an active prismatic joint denoted by $P$ that extend and retract the leg. The movable platform has six degrees of freedom, three translational and three rotational motions. Fig. 1 shows the Stewart-Gough manipulator.

\section{A. Structural modeling}

Two coordinate frames $\{P\}$ and $\{B\}$ are attached to the movable and fixed base respectively. The vector $\mathbf{b}_{i}=$ $\left[\begin{array}{lll}b_{i x} & b_{i y} & b_{i z}\end{array}\right]^{T}$ describes the position of the reference point $B_{i}$ with respect to the frame $\{B\}$; In the same way, the vector $\mathbf{p}_{i}=\left[\begin{array}{lll}p_{i x} & p_{i y} & p_{i z}\end{array}\right]^{T}$ describes the position of the reference point $P_{i}$ with respect to the reference frame $\{P\}$ (see Fig. 2).

$$
\begin{aligned}
\mathbf{b}_{i} & =\left[\begin{array}{lll}
r_{b} \cos \left(\psi_{i}\right) & r_{b} \sin \left(\psi_{i}\right) & 0
\end{array}\right]=\left[\begin{array}{lll}
b_{i x} & b_{i y} & b_{i z}
\end{array}\right] \\
\mathbf{p}_{i} & =\left[\begin{array}{llll}
r_{p} \cos \left(\Psi_{i}\right) & r_{p} \sin \left(\Psi_{i}\right) & 0
\end{array}\right]=\left[\begin{array}{lll}
p_{i x} & p_{i y} & p_{i z}
\end{array}\right]
\end{aligned}
$$




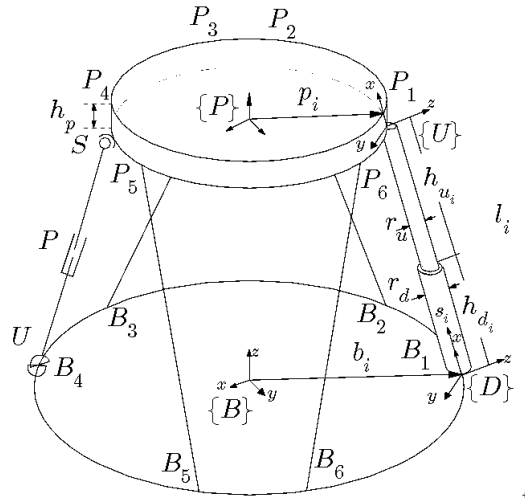

Fig. 1. Stewart-Gough robot.

where

$$
\begin{array}{clc}
\psi_{i}=\frac{i \pi}{3}-\frac{\phi_{b}}{2} & \Psi_{i}=\frac{i \pi}{3}-\frac{\phi_{p}}{2} & i=1,3,5 \\
\psi_{i}=\psi_{i-1}+\phi_{b} & \Psi_{i}=\Psi_{i-1}+\phi_{p} & i=2,4,6
\end{array}
$$

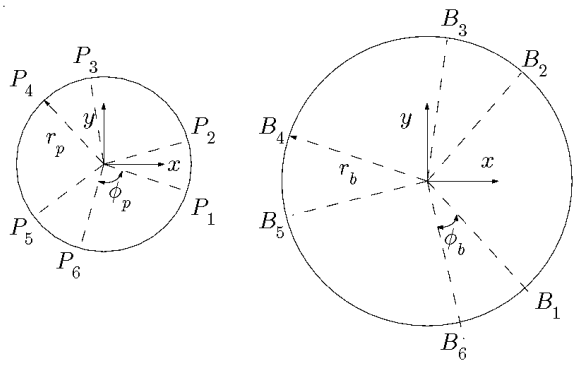

Fig. 2. Fixed base and movable platform.

The Stewart-Gough manipulator geometry was defined with two coplanar set of vectors: $\mathbf{b}$ and $\mathbf{p} ; \mathbf{b}$ corresponds to the fixed base and $\mathbf{p}$ to the movable platform [1]. According to Eq. (1), the Stewart-Gough mechanism can be defined by five structure design variables: $r_{b}$ is the radius of the fixed base, $r_{p}$ is the radius of the movable platform, $\phi_{b}$ is the spacing angle of the vectors $\mathbf{b}_{i}, \phi_{p}$ is the spacing angle of the vectors $\mathbf{p}_{i}$. Finally, $s$ sets the length of the lower member as function of total length of the leg, thus the length of the upper member is defined as $h_{d_{i}}=s_{i} l_{i}$ and the length of the lower member is $h_{u_{i}}=\left(1-s_{i}\right) l_{i}$. Consequently, the structure of mechanism can be parametrized by the vector $\boldsymbol{\lambda}_{s} \in \mathbb{R}^{5 \times 1}$.

$$
\boldsymbol{\lambda}_{s}=\left[\begin{array}{lllll}
r_{p} & \phi_{p} & r_{b} & \phi_{b} & s
\end{array}\right]^{T}
$$

\section{B. Dynamic Model}

The dynamic equations for the 6-UPS Stewart-Gough manipulator were derived in closed form through the NewtonEuler approach by [4].

$$
\mathbf{J}^{-1} \mathbf{M}(\mathbf{q}) \mathbf{J}^{-T} \ddot{\mathbf{q}}+\mathbf{J}^{-1}\left[\boldsymbol{\eta}(\mathbf{q}, \dot{\mathbf{q}})-\boldsymbol{M}(\mathbf{q}) \mathbf{J}^{-T} \mathbf{u}\right]=\mathbf{f}
$$

where,
- $\mathbf{f}=\left[\begin{array}{lll}f_{1} & \ldots & f_{6}\end{array}\right]^{T} \in \mathbb{R}^{6 \times 1}$ is the actuator force vector.

- $\mathbf{J}=\left[\begin{array}{ccc}\mathbf{s}_{1} & \ldots & \mathbf{s}_{6} \\ \mathbf{p}_{1} \times \mathbf{s}_{1} & \ldots & \mathbf{p}_{6} \times \mathbf{s}_{6}\end{array}\right] \in \mathbb{R}^{6 \times 6}$ is the Jacobian matrix. $\mathbf{s}_{i}$ is the unit vector along each leg, for $i=1, \ldots, 6$ (Fig. 1).

- $\quad \mathbf{q}=\left[\begin{array}{lll}l_{1} & \ldots & l_{6}\end{array}\right]^{T} \in \mathbb{R}^{6 \times 1}$ is the leg length vector.

- $\ddot{\mathbf{q}}=\left[\begin{array}{lll}\ddot{l}_{1} & \ldots & \ddot{l}_{6}\end{array}\right]^{T} \in \mathbb{R}^{6 \times 1}$ is the leg acceleration vector.

- $\quad \mathbf{M}=\mathbf{M}_{\text {plat }}+\sum_{i=1}^{6} \mathbf{M}_{i} \in \mathbb{R}^{6 \times 6}$ is the total inertia matrix which considers the inertia of the legs and the movable platform.

- $\boldsymbol{\eta}=\boldsymbol{\eta}_{\text {plat }}+\sum_{i=1}^{6} \boldsymbol{\eta}_{i} \in \mathbb{R}^{6 \times 1}$ is the Coriolis, gravitation, and centrifuge force vector of the movable platform and each leg.

- $\mathbf{u} \in \mathbb{R}^{6 \times 1}$ is an expression related to the acceleration of the legs.

Additional details of the formulation of the dynamic equations can be obtained in [4]. Eq. (3) can be written in a simplified way:

$$
\mathbf{A}(\mathbf{q}) \ddot{\mathbf{q}}+\mathbf{h}(\mathbf{q}, \dot{\mathbf{q}})=\mathbf{f}
$$

where $\mathbf{A}(\mathbf{q})=\mathbf{J}^{-1} \mathbf{M}(\mathbf{q}) \mathbf{J}^{-T}$ and $\mathbf{h}(\mathbf{q}, \dot{\mathbf{q}})=\mathbf{J}^{-1}[\boldsymbol{\eta}(\mathbf{q}, \dot{\mathbf{q}})-$ $\left.\boldsymbol{M}(\mathbf{q}) \mathbf{J}^{-T} \mathbf{u}\right]$.

The inertial properties of the movable platform and the six legs are defined as function of the structure variables of Eq. (2). The geometric shape of the rigid bodies of the Stewart-Gough mechanism is defined as cylinders (see Fig. 1). The inertia matrix and center of mass are defined based on the structure variables $\boldsymbol{\lambda}_{s}$ and the density of each rigid body. The center of mass of the movable platform is attached to the coordinate frame $\{P\}$. The inertia matrix of the lower and upper member of the legs is defined with respect to the coordinate frames $\{U\}$ and $\{D\}$ and the centers of mass are $\mathbf{r}_{u}=\left[\begin{array}{lll}-h_{u} & 0 & 0\end{array}\right]^{T}$ and $\mathbf{r}_{d}=\left[\begin{array}{lll}-h_{d} & 0 & 0\end{array}\right]^{T}$, respectively. The following parameters should be defined in order to describe the inertia of all rigid bodies as function of the structural parameters $\lambda_{s}$ : thickness of the movable platform $h_{p}$, radius of the upper $r_{u}$ and lower $r_{l}$ members of the legs, density of the material of the movable platform $\rho_{p}$ and legs $\rho_{l}$.

\section{Tracking position control}

Six independent joint space PD controllers are used to track a desired trajectory (Fig. 3).

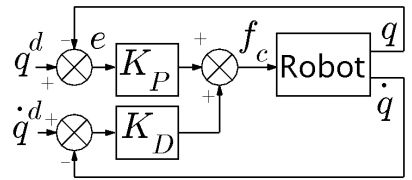

Fig. 3. Joint space PD controller of Stewart-Gough robot.

Assuming that the desired trajectory for each actuator is specified with the desired joint space position $\mathbf{q}^{d}$ and velocity $\dot{\mathbf{q}}^{d}$, the control law is:

$$
\mathbf{f}_{c}=\mathbf{K}_{P}\left(\mathbf{q}^{d}-\mathbf{q}\right)+\mathbf{K}_{D}\left(\dot{\mathbf{q}}^{d}-\dot{\mathbf{q}}\right)
$$


where, $\mathbf{K}_{P}=\operatorname{diag}\left(k_{p_{i}}, \ldots, k_{p_{i}}\right), \mathbf{K}_{D}=\operatorname{diag}\left(k_{d_{i}}, \ldots, k_{d_{i}}\right)$, for $i=1, \ldots, 6$. Assuming that the proportional and derivative gains of the six PD controllers are equal, the tracking position controller can be parametrized by the vector $\boldsymbol{\lambda}_{c} \in \mathbb{R}^{2 \times 1}$.

$$
\boldsymbol{\lambda}_{c}=\left[\begin{array}{ll}
k_{p} & k_{d}
\end{array}\right]^{T}
$$

Several methods to tune the PD controllers of robot manipulators have concluded that positive gains stabilize robot [12].

The closed-loop dynamics of the robot with the PD controller is formulated by using the state space formalism.

$$
\dot{\mathbf{x}}=f\left(\mathbf{x}, \mathbf{x}^{d}, \boldsymbol{\lambda}, t\right)=\left[\begin{array}{c}
\dot{\mathbf{q}} \\
-\mathbf{A}^{-1}(\mathbf{q}) \mathbf{h}(\mathbf{q}, \dot{\mathbf{q}})
\end{array}\right]+\left[\begin{array}{c}
0 \\
-\mathbf{A}^{-1}(\mathbf{q})
\end{array}\right] \mathbf{f}_{c}
$$

with $\dot{\mathbf{x}}=\left[\begin{array}{ll}\dot{\mathbf{q}} & \ddot{\mathbf{q}}\end{array}\right]^{T} \in \mathbb{R}^{12 \times 1}, \mathbf{x}^{d}=\left[\begin{array}{ll}\mathbf{q}^{d} & \dot{\mathbf{q}}^{d}\end{array}\right]^{T} \in \mathbb{R}^{12 \times 1}$.

and $\lambda \in \mathbb{R}^{7 \times 1}$ is the vector of the structure-control design variables.

$$
\boldsymbol{\lambda}=\left[\begin{array}{ll}
\boldsymbol{\lambda}_{s} & \boldsymbol{\lambda}_{c}
\end{array}\right]^{T}=\left[\begin{array}{lllllll}
r_{p} & \phi_{p} & r_{b} & \phi_{b} & s & k_{p} & k_{d}
\end{array}\right]^{T}
$$

As seen in Eq. (7), the closed loop dynamics of the StewartGough robot depends on the structure-control design variables.

\section{Objective function}

Performance criteria based on Jacobian matrix have been widely used in the optimal design to improve the dexterity and accuracy of serial robots [13]. By using Jacobian matrix analysis it is possible to determine the singularity loci of Stewart-Gough platform. The 6-UPS Stewart-Gough platform is a spatial mechanism. The conventional Jacobian matrix expresses a coupled relation of both translational and rotational motions. The elements of the conventional Jacobian matrix have nonhomogenous physical units. Therefore, the use of performance indices such as the condition number of the Jacobian matrix may lead to a lack of physical meaning [2].

kinetostatic performance indices indicate when the parallel robot is closed to a singular configuration as an alternative to indices based on the Jacobian matrix [14]. The singularity zones in the workspace can be characterized with the aid of kinetostatic performance indices. When the parallel manipulator is close to the singularity zone, they lose their stiffness and their quality of motion transmission, this affects the position accuracy of the robot.

Furthermore, it has been demonstrated that the motion through such singularity loci is feasible and the singularities can also be examined based on the dynamics of the robot. Nevertheless, at this specific condition during motion the position accuracy decreases significantly [15]. Consequently, performance criterion based on the closed-loop dynamics are suitable for the optimal design of the Stewart-Gough robot. The tracking position error of PD position controller in Eq. (5) is selected since the objective of this contribution is to improve the position tracking accuracy of the robot.

Integrating Eq. (7) over an imposed trajectory $\mathbf{x}^{d}$ leads to the actual closed loop position of the robot. Thus, the tracking position error of the six legs is $\mathbf{e}(\boldsymbol{\lambda}))=\left(\mathbf{q}^{d}-\mathbf{q}\right) \in \mathbb{R}^{6 \times 1}$. The objective function to be minimized $J$ is the total tracking error evaluated by means of the Root Mean Square Error (RMSE) of the six legs:

$$
J=R M S E(\mathbf{e}(\boldsymbol{\lambda}))=\frac{1}{6} \sum_{i=1}^{6} \sqrt{e_{i}^{T} e_{i}}
$$

\section{SENSITIVITY ANALYSIS}

The previous sections presented the robot modeling, the parametrization of the structure-control design variables and the objective function for the optimal design. It would be interesting to evaluate the effect of each variable independently on the variation of the position accuracy of the robot. Additionally, the sensitivity analysis allows to understand the effect of each design variable within the search space to meet the optimum criterion.

The sensitivity analysis aims at determining the influence of each structure-control variable of Eq. (8) on the dynamic response. Consequently, this analysis allows to indicate the degree of influence of each variable on the variation of the dynamic response, specifically on the position accuracy of the robot.

Among the various methods used to analyze the sensitivity, the variance-based sensitivity analysis decomposes the variance of the output of the model into fractions which are associated with the variation of each variable [16]. This method allows to quantify the effect of the variation of an individual variable on the dynamic response of the robot by means of a probabilistic framework based on the Monte Carlo Simulation method. Additionally, this method copes with nonlinear models, which is suitable to quantify the sensitivity of the robot.

Considering the model under the form $y=f(\mathbf{w})$, where $y$ a scalar output and $\mathbf{w}=\left[\begin{array}{lll}w_{1} & \ldots & w_{k}\end{array}\right]^{T} \in \mathbb{R}^{k \times 1}$ is a vector of $k$ design variables. These variables are considered as independently and uniformly distributed within the unit hypercube, i.e., $w_{i} \in[0,1]$ for $i=1, \ldots, k . f(\mathbf{w})$ is decomposed:

$y=f(\mathbf{w})=f_{0}+\sum_{i=1}^{k} f_{i}\left(w_{i}\right)+\sum_{i<j}^{k} f_{i j}\left(w_{i}, w_{j}\right)+\cdots+f_{12 \ldots, k}$

The decomposition of the variance expression is [17]:

$$
V(y)=\sum_{i=1}^{k} V_{i}+\sum_{i<j}^{k} V_{i j}+\cdots+V_{12 \ldots k}
$$

where, $V_{i}=V_{w_{i}}\left(E_{\mathbf{w}_{\sim i}}\left(y \mid w_{i}\right)\right), V_{i j}=V_{w_{i j}}\left(E_{\mathbf{w}_{\sim i j}}\left(y \mid w_{i j}\right)\right)$, and so on. A variance based first order effect for a generic design variable $w_{i}$ is:

$$
V_{w_{i}}\left(E_{\mathbf{w}_{\sim i}}\left(y \mid w_{i}\right)\right)
$$

where $w_{i}$ is the $\mathrm{i}$-th variable and $\mathbf{w}_{\sim i}$ denotes the matrix of all variable except $w_{i}$. The meaning of the inner expectation operation is that the mean of $y$ is taken over all possible values $\mathbf{w}_{\sim i}$ while keeping $w_{i}$ fixed. The associated sensitivity measure denominated first-order sensitivity index is defined as:

$$
s_{i}=\frac{V_{w_{i}}\left(E_{\mathbf{w}_{\sim i}}\left(y \mid w_{i}\right)\right)}{V(y)}
$$


$s_{i}$ states the effect of the variation of $w_{i}$ only, however divided by the variation in other variables. Nevertheless, the total effect-index $s_{T i}$ measures the contribution to the output variance of $w_{i}$, including all the effects of its interactions with any other input variable.

$$
s_{T i}=\frac{E_{\mathbf{w}_{\sim i}}\left(V_{w_{i}}\left(y \mid w_{\sim i}\right)\right)}{V(y)}=1-\frac{V_{\mathbf{w}_{\sim i}}\left(E_{w_{i}}\left(y \mid w_{\sim i}\right)\right)}{V(y)}
$$

The Monte Carlo Simulation combined with the Latin Hypercube sampling [18] is used to calculate the total-effect indices. The total number of model evaluation to compute the totalsensitivity index is $N=n_{s}(k+1)$, where $n_{s}$ is the number of the Monte Carlo samples [16].

\section{OPTIMIZATION PROBLEM}

In this contribution, the optimal design aims at selecting the optimal structure-control design variables according to dynamic and geometric constraints. The optimization problem is solved to minimize position tracking error over a required workspace trajectory. For practical purposes, a required workspace trajectory is defined. The related joint-space position reference trajectory $\mathbf{q}^{d}$ is obtained by means of the inverse kinematic model. The joint-space velocity reference $\dot{\mathbf{q}}^{d}$ is proportional to the workspace velocity as stated by [4].

Thus, the optimization problem to select the structurecontrol design variables $\boldsymbol{\lambda}$ of the parallel robot is given by

$$
\begin{aligned}
& \min _{\boldsymbol{\lambda}}\{J=R M S E(e(\boldsymbol{\lambda}))\} \\
& \text { subject to } \\
& r_{p}, r_{b} \in\left[r_{\text {min }}, r_{\text {max }}\right] \\
& \phi_{p} \in\left[\phi_{\min _{p}}, \phi_{\max _{p}}\right], \phi_{b} \in\left[\phi_{\text {min }_{b}}, \phi_{\max _{b}}\right] \\
& s \in\left[s_{\min }, s_{\max }\right] \\
& k_{p} \in\left[k_{p_{\min }}, k_{p_{\max }}\right], k_{v} \in\left[k_{v_{\min }}, k_{v_{\max }}\right] \\
& \mathbf{f}_{c} \in\left[\mathbf{f}_{\text {min }_{c}}, \mathbf{f}_{\text {max }_{c}}\right] \\
& \forall \mathbf{q} \in \mathbf{q}^{d}, \forall \dot{\mathbf{q}} \in \dot{\mathbf{q}}^{d}
\end{aligned}
$$

Metaheuristic algorithms for optimization have been successfully applied to nonlinear and constraint problems in order to find the global minima. Thus, this optimization problem is solved by using Genetic Algorithm [10] and Differential Evolution [11].

\section{Simulation Results}

This section presents the results of the sensitivity analysis and the optimal design. For the proposed optimization problem, the sensitivity analysis helps to evaluate the effect of the design variables within the search space on the objective function.

As presented in section II-B the model parameters of Table I should be imposed to define completely the parameters of dynamic equation of the robot as function the structurecontrol design variables $\boldsymbol{\lambda}$. The simulations were implemented using MATLAB.
TABLE I. MOdel PARAMETERS

\begin{tabular}{|l||l||}
\hline Parameter & Value \\
\hline$\rho_{p}$ & $7874 \mathrm{~kg} / \mathrm{m}^{3}$ \\
\hline$\rho_{l}$ & $2697 \mathrm{~kg} / \mathrm{m}^{3}$ \\
\hline$r_{u}$ & $0.03 \mathrm{~m}$ \\
\hline$r_{d}$ & $0.03 \mathrm{~m}$ \\
\hline
\end{tabular}

\section{A. Sensitivity analysis}

The sensitivity analysis was performed based on the model of the controlled Stewart-Gough robot presented on section II. This analysis is performed over an imposed circular workspace trajectory (see Fig. 5), nevertheless any other trajectory could be considered. The total effect-indices of the structure-control design variables $\boldsymbol{\lambda}$ of Eq. (8) are computed by using the variance-based sensitive analysis presented on section III.

In order to perform the sensitivity analysis, each design variable of $\boldsymbol{\lambda}$ was modeled as a normal distributed random variable. The mean $\bar{\lambda}_{i}$ and standard deviation $\sigma_{i}$ were selected in order to establish the dispersion of each random variable (in Table II) within the search space of the optimization problem defined in Eq. (16).

TABLE II. PARAMETERS OF NORMAL RANDOM VARIABLES.

\begin{tabular}{|l||l|l|l|l|l|l|l||}
\hline & $r_{p}[\mathrm{~m}]$ & $\phi_{p}\left[^{\circ}\right]$ & $r_{b}[\mathrm{~m}]$ & $\phi_{b}\left[^{\circ}\right]$ & $s$ & $k_{p}$ & $k_{v}$ \\
\hline $\bar{\lambda}_{i}$ & 0.4 & 90 & 0.4 & 30 & 0.5 & 80000 & 400 \\
\hline$\sigma_{i}$ & 0.0667 & 10 & 0.0667 & 10 & 0.0667 & 26667 & 133.3 \\
\hline
\end{tabular}

The number of computation of the Monte Carlo samples required to perform the sensitivity analysis was fixed at $n_{s}=100$ to ensure an accurate solution. Considering $k=7$ variables, the total number of model evaluations is $N=800$.

The total effect-indices of the design variables $\boldsymbol{\lambda}$ for the circular trajectory are showed in Fig. 4. As seen, the position accuracy is more sensitive to the proportional gain $k_{p}$ of PD position controller than the other variables. This is expected since the position error is proportional to this gain. However, among the structure variables the radius of the movable platform $r_{p}$ exhibits a significant sensitivity.

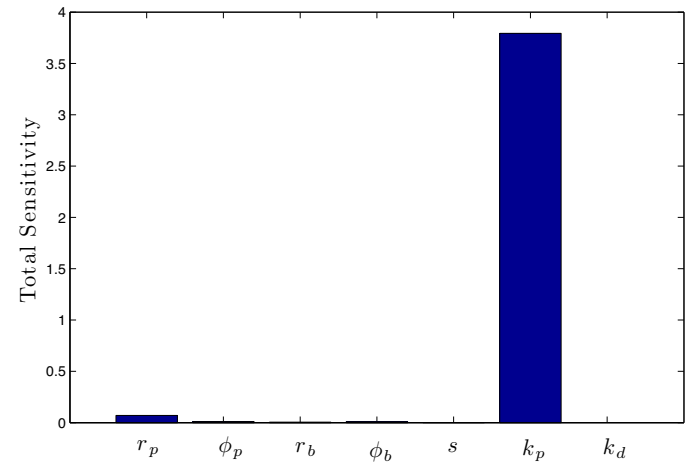

Fig. 4. Total effect-indices over the reference trajectory.

In order to determine the sensitivity of the structure variables for the same circular trajectory of the previous analysis, the control variables are considered as constant. The constant 
values of control are their means $\bar{k}_{p}$ and $\bar{k}_{v}$ of Table II. Figure 5 shows the circular reference trajectory and the trajectories obtained by using the structure variables of the Monte Carlo samples. This indicates that, even with constant controller gains the position accuracy is very sensitive to the structural design variables.

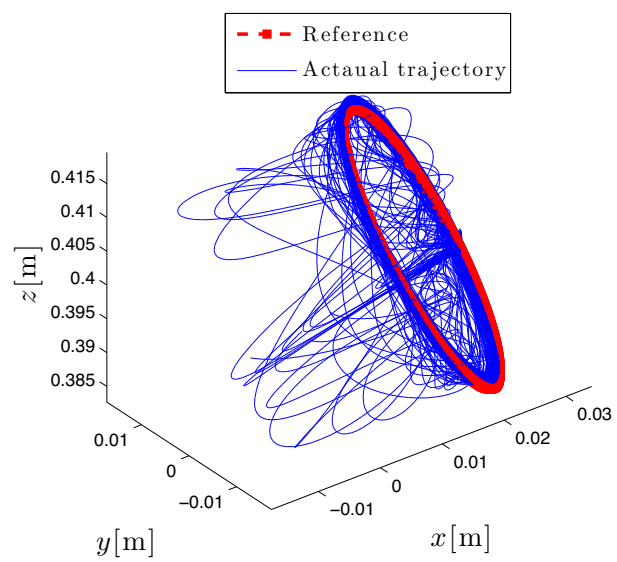

Fig. 5. Sensitivity analysis: reference and actual trajectory.

The total effect-indices of structure design variables for the circular trajectory are showed in Fig. 6. This demonstrates that the position accuracy is high sensitive to the radius of the movable platform as seen in the sensitivity analysis of Fig. 4. The radius of the fixed base, the spacing angles of the movable platform and fixed base show a considerable sensitivity in view of the fact that the Jacobian matrix depends on these design variables. The length of the upper and lower members of the legs is slightest sensitive, hence the variations in the length of member have minor effects on position accuracy.

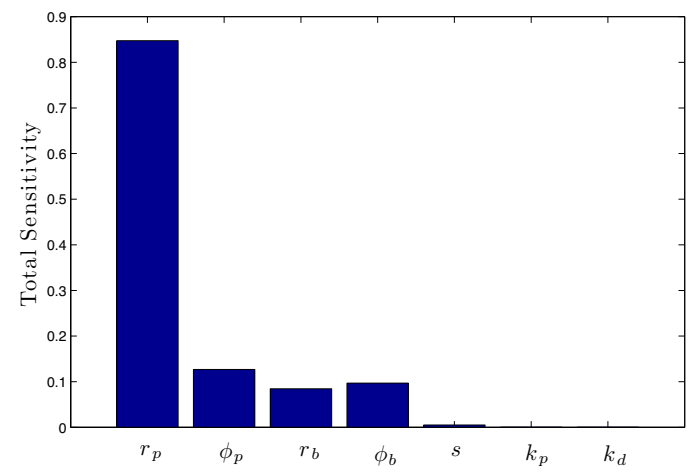

Fig. 6. Total effect-indices with fixed controller gains.

\section{B. Optimization}

The structure-control design variables of vector $\lambda$ of Eq. (8) are optimized for obtaining the minimum RMS error over an imposed workspace trajectory (see Fig. 5), i.e. to maximize the position accuracy. The constraints of the design variables are limited by manufacturing tolerances and the maximum power of the actuators. The optimization problem is formulated as follows:

$$
\begin{aligned}
& \min _{\boldsymbol{\lambda}}\{J=R M S E(e(\boldsymbol{\lambda}))\} \\
& \text { subject to } \\
& r_{p}, r_{b} \in[0.2 \mathrm{~m}, 0.6 \mathrm{~m}] \\
& \phi_{p} \in\left[60^{\circ}, 120^{\circ}\right], \phi_{b} \in\left[0^{o}, 60^{\circ}\right] \\
& s \in[0.3,0.7] \\
& k_{p} \in[0,160000], k_{v} \in[0,800] \\
& \mathbf{f}_{c} \in[-100 \mathrm{~N}, 100 \mathrm{~N}] \\
& \forall \mathbf{q} \in \mathbf{q}^{d}, \forall \dot{\mathbf{q}} \in \dot{\mathbf{q}}^{d}
\end{aligned}
$$

After some preliminary simulations, the tuning parameters used in the GA and DE optimization algorithm are presented in Table III.

TABLE III. PARAMETERS USED IN THE GA AND DE ALGORITHMS

\begin{tabular}{|l||l|l||}
\hline Parameter & GA & DE \\
\hline Max. Generation number & 100 & 100 \\
\hline Population size & 70 & 70 \\
\hline Crossover probability & 0.5 & 0.5 \\
\hline Perturbation rate & - & 0.8 \\
\hline Strategy & - & DE/rand/1/bin \\
\hline Mutation rate & 0.08 & - \\
\hline
\end{tabular}

In order to evaluate the solution of the optimization problem of Eq. (16), the evolution of the objective function along the generations using the GA and DE optimization algorithm is presented in Fig. 7. When GA optimization method is used, the objective function converges after 56 generations and its value is $\operatorname{RMSE}(\mathbf{e})=0.1530 \times 10^{-3} \mathrm{~m}$, the objective function was computed 2925 times among which 437 exhibited a singularity condition. When DE optimization method is used, the objective function converges after 51 generations and its value is $\operatorname{RMSE}(\mathbf{e})=0.1451 \times 10^{-3} \mathrm{~m}$, the objective function was computed 3543 times among which 1385 exhibited a singularity condition.

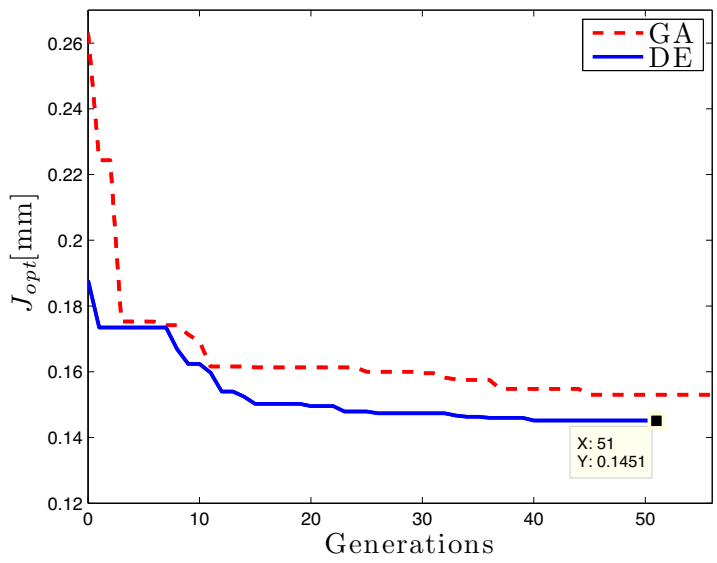

Fig. 7. Objective function evolution using GA and DE.

As seen in Table IV, the optimization results are similar using both optimization algorithms. Even though, the objective 
function was computed fewer times by using GA optimization, however, the solution obtained using DE algorithm exhibits a better solution by using the tuning parameters of Table III.

TABLE IV. OPTIMIZATION RESULTS, DESIGN VARIABLES

\begin{tabular}{|l||l|l|l|l|l|l|l||}
\hline & $r_{p}[\mathrm{~m}]$ & $\phi_{p}\left[{ }^{\circ}\right]$ & $r_{b}[\mathrm{~m}]$ & $\phi_{b}\left[^{\circ}\right]$ & $s$ & $k_{p}$ & $k_{v}$ \\
\hline GA & 0.2468 & 103.372 & 0.3232 & 42.2860 & 0.7000 & 160000 & 62 \\
\hline DE & 0.2379 & 95.3968 & 0.2918 & 32.2675 & 0.6962 & 159610 & 250 \\
\hline
\end{tabular}

The proportional gain of the controller reached its maximum feasible value. This is expected since magnitude of this variable is proportional to the position accuracy. The structure design variables over the circular reference trajectory were selected by the optimization algorithms to avoid configurations closed to singularity zones taking into account the closed-loop dynamics of the robot. Consequently, the optimized structure variables minimize the degradation of position accuracy during the motion.

An additional simulation was considered in order to evaluate the position accuracy with the design variables obtained by DE algorithm (Table IV). The circular reference trajectory of the Fig. 5 is considered to assess the workspace error obtained with the initial $\boldsymbol{\lambda}_{0}$ and optimized $\boldsymbol{\lambda}_{\text {opt }}$ set of structurecontrol design variables. As expected, Fig. 8 shows that the workspace error is minimized by using the optimal structurecontrol design variables obtained by DE optimization.

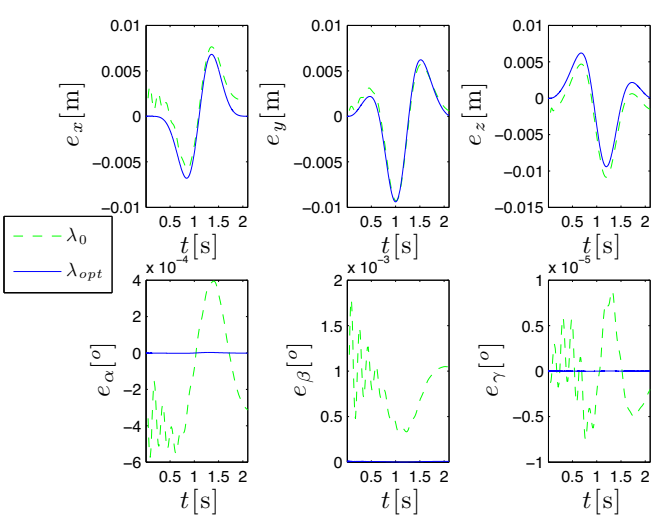

Fig. 8. Workspace error with .

\section{CONCLUSION}

This contribution presented an optimal design procedure, minimizing the tracking accuracy over an imposed trajectory, to find simultaneously the structure-control design variables of a Stewart-Gough robot. This methodology allows to find the optimal performance of the robot considering dynamic and kinematic properties simultaneously.

The sensitivity analysis demonstrated the great influence of structure variables of the mechanism on the position accuracy of robot. Metaheuristic optimization algorithms has shown to be a robust optimization tool to find optimal structure-control design variables for this optimal design problem.

\section{ACKNOWLEDGMENT}

The authors express their acknowledgements to the Graduate Program in Mechanical Engineering of the Federal Technological University of Paraná funded by CAPES.

\section{REFERENCES}

[1] F. Lara-Molina, J. Rosario, and D. Dumur, "Multi-objective optimization of stewart-gough manipulator using global indices," in Advanced Intelligent Mechatronics (AIM), 2011 IEEE/ASME International Conference on, pp. 79-85, 2011.

[2] J. P. Melet, "Jacobian, manipulability, condition number, and accuracy of parallel robots," Journal of Mechanical Design, vol. 128, no. 199206, 2006.

[3] J. Ryu and J. Cha, "Volumetric error analysis and architecture optimization for accuracy of hexaslide type parallel manipulators," Mechanism and Machine Theory, vol. 38, no. 3, pp. 227-240, 2003.

[4] B. Dasgupta and T. S. Mruthyunjaya, "Closed-form dynamic equations of the general stewart platform through the Newton-Euler approach," Mechanism and Machine Theory, vol. 33, no. 7, pp. 993-1012, 1998.

[5] F. Lara-Molina, J. Rosário, and D. Dumur, "Robust generalized predictive control of stewart-gough platform," in Robotics Symposium, 2011 IEEE IX Latin American and IEEE Colombian Conference on Automatic Control and Industry Applications (LARC), pp. 1-6, 2011.

[6] F. A. Lara-Molina, J. M. Rosário, D. Dumur, and P. Wenge, "Robust generalized predictive control of the orthoglide robot," Industrial Robot: An International Journal, vol. 41, no. 3, pp. 275-285, 2014.

[7] M. G. Villarreal-Cervantes, C. A. Cruz-Villar, and J. Alvarez-Gallegos, "Structure-control mechatronic design of the planar $5 \mathrm{r}$ 2dof parallel robot," in Structure-Control Mechatronic Design of the Planar Proceedings of the 2009 IEEE International Conference on Mechatronics, 2009.

[8] M. M. da Silva and L. A. M. Gonçalves, "Mechatronic design concept and its application to pick-and-place robotic systems," Journal of the Brazilian Society of Mechanical Sciences and Engineering, vol. 35, pp. 31-40, 2013.

[9] M. Villarreal-Cervantes, C. Cruz-Villar, J. Alvarez-Gallegos, and E. Portilla-Flores, "Robust structure-control design approach for mechatronic systems," Mechatronics, IEEE/ASME Transactions on, vol. 18, no. 5, pp. 1592-1601, 2013.

[10] S. Sivanandam and S. Deepa, Genetic Algorithm Optimization Problems. Springer, 2008.

[11] K. V. Price, R. M. Storn, and J. A. Lampinen, Differential evolution a practical approach to global optimization. Springer-Verlag, 2005.

[12] W. Yu, X. Li, and R. Carmona, "A novel PID tuning method for robot control," Industrial Robot: An International Journal, vol. 40, no. 6, pp. 574-582, 2013.

[13] C. Gosselin and J. Angeles, "A global performance index for the kinematic optimization of robotic manipulators," Journal of Mechanical Design, vol. 113, no. 3, pp. 220-226, 1991.

[14] D. Zhang, F. Xi, C. M. Mechefske, and S. Y. Lang, "Analysis of parallel kinematic machine with kinetostatic modelling method," Robotics and Computer-Integrated Manufacturing, vol. 20, no. 2, pp. 151-165, 2004.

[15] D. N. Nenchev, S. Bhattacharya, and M. Uchiyama, "Dynamic analysis of parallel manipulators under the singularity-consistent parameterization," Robotica, vol. 15, no. 04, pp. 375-384, 1997.

[16] A. Saltelli, M. Ratto, T. Andres, F. Campolongo, J. Cariboni, D. Gatelli, M. Saisana, and S. Tarantola, Global Sensitivity Analysis: The Primer. John Wiley \& Sons Chichester, England, 2008.

[17] I. M. Sobol', “On sensitivity estimation for nonlinear mathematical models," Matematicheskoe Modelirovanie, vol. 2, no. 1, pp. 112-118, 1990.

[18] A. Florian, "An efficient sampling scheme: updated latin hypercube sampling," Probabilistic engineering mechanics, vol. 7, no. 2, pp. 123 130, 1992. 